\title{
The relevance of conductive additive addition methodology for optimizing the performance of electrodes based on carbon xerogels in aqueous supercapacitors
}

\author{
María Canal-Rodríguez, J. Angel Menéndez, Miguel A. Montes-Morán, Ana Arenillas* \\ Instituto Nacional de carbón, CSIC, Francisco Pintado Fe, 26,33011, Oviedo, Spain \\ aapuente@incar.csic.es
}

\begin{abstract}
Electrodes for supercapacitors are commercially produced by mixing active material with a conductive additive and a binder. However, with some polymers such as carbon xerogels the conductive additives can be added during their synthesis in order to enhance interactions between the components of the electrode. In this work the performance in aqueous supercapacitors of a carbon xerogel and carbon black or graphene added during and post synthesis is compared. It was observed that, the more integrated the additive is within the carbon xerogel polymeric structure, the greater the differences in the final performance of the hybrid materials, being the sample with graphene oxide added during the synthesis of the organic gel the one with the best performance throughout the whole range of current densities studied.
\end{abstract}

Keywords: carbon xerogels, electrical conductivity, carbon black, graphene oxide, supercapacitors. 


\section{Introduction}

Supercapacitors are electrochemical devices that are attracting a great deal of attention among the scientific community. However, to ensure a more extensive use of these devices it is necessary to increase their energy density, without impairing their power density. One possible way to achieve this is to develop advanced electrode materials with an optimal combination of surface chemistry, adequate porosity and high electrical conductivity [1]. However achieving the right combination is not an easy task, as these properties are interdependent and sometimes opposing.

Commercial supercapacitors are made using activated carbons, which are usually just microporous carbons. Sometimes the microporosity may be inaccessible due to diffusional problems of the electrolyte used. Therefore a combination of micropores and the so-called feeder pores (i.e. narrow mesopores) is required [2]. On the other hand, surface oxygen functionalities may increase the interaction of aqueous electrolyte/electrode, although such functionalities may have a negative effect on the electrical conductivity of the active materials, which is one of the most important properties that the electrodes must have.

In order to increase this conductivity, the physical mixture of the active material with a conductive additive and a binder is normally the procedure used for building the electrodes [3]. This is the only possible solution in the case of active carbons. However, synthetic polymers also offer several advantages: (i) high purity materials; (ii) high control of the porosity (micro and mesoporosity); and (iii) conductive additives can be added during their own synthesis. The latter would probably be traduced in a better performance than mixing the components physically, as it is usually performed. 
In this work, the two different approaches to manufacturing electrodes (i) adding the conductive additive to the active material by physically mixing and (ii) introducing the conductive additive during the synthesis of the active material precursor, are compared. The ability of these two approaches to improve the performance of the electrodes in supercapacitors was also analyzed under different operating conditions.

\section{Experimental}

\subsection{Synthesis of the materials studied}

An activated carbon xerogel (AX) was obtained by the one-step activation/carbonization of an organic xerogel precursor obtained by the polycondensation of resorcinol (R) and formaldehyde $(\mathrm{F})$, in a molar ratio $\mathrm{R} / \mathrm{F}$ of 0.5 , in water (dilution ratio of 5.7) and initial $\mathrm{pH}$ of 6.5. Detailed description of the synthesis procedure of this carbon material can be found elsewhere [2, 4].

The two conductive materials used in this study were a carbon black (CB) from Cabot Corporation, and a graphene oxide (GO) supplied by Applynano Solutions. These two additives were selected because (i) both of them can be prepared in stable aqueous suspensions, to facilitate their introduction during the synthesis of the RF xerogel and

(ii) $\mathrm{CB}$ has a high electrical conductivity (i.e. $1700 \mathrm{~S} \mathrm{~m}^{-1}$ as measured by the four point probe technique [5]) and although GO by itself does not have a high conductivity, after the treatment at $1000^{\circ} \mathrm{C}$ is expected to produce a reduced graphene oxide (RGO) with excellent conductivity [6].

The two additives are introduced in two different stages: (i) during the synthesis of the RF xerogel, when the water normally used as solvent is replaced by a $0.5 \mathrm{wt} \%$ suspension of each additive [7], to obtain the samples AXCB and AXGO; and (ii) post- 
synthesis, when the activated carbon xerogel $\mathrm{AX}$ is physically mixed with the $\mathrm{CB}$ or the reduced graphene oxide ( $R G O)$ to produce the samples $A X+C B$ and $A X+R G O$, respectively.

In order to reduce the variables involved in the performance of the supercapacitors, the activation/carbonization step are adjusted to obtain materials with similar BET areas (ca. $1600 \mathrm{~m}^{2} \mathrm{~g}^{-1}$ ). Moreover, the quantity of conductive additives added is also adjusted so that the final samples have ca. $2 \mathrm{wt} \%$ of additive. A commercial carbon (YP-50F from Kuraray) was also evaluated for comparative purposes.

Disc-shape electrodes of the samples were manufactured by mixing $90 \mathrm{wt} \%$ of each sample with $10 \mathrm{wt} \%$ of polytetrafluoroethylene (PTFE) as a binder [8]. The electrodes were punched, from the film obtained from this mixture, with a circular shape, diameter of $10 \mathrm{~mm}$, thickness of ca. 100 microns and weight of ca. $5 \mathrm{mg}$. These electrodes were used for electrical conductivity measurements and electrochemical characterization.

\subsection{Characterization techniques used}

The porous characteristics were evaluated on the basis of $\mathrm{N}_{2}$ adsorption-desorption isotherms at $77 \mathrm{~K}$ (Micromeritics Tristar 3020).

Temperature-programmed desorption (TPD) experiments were performed on a Micromeritics AutoChem II analyzer. The quantity of $\mathrm{CO}$ and $\mathrm{CO}_{2}$ desorbed was monitored on a mass spectrometer (OmniStar Pfeiffer).

The electrical conductivity of the materials was obtained by applying the Van der Pauw equation using the four-point probe technique (FPP) (model SR-4-6L, Everbeing) [5].

The electrochemical characterization was performed using a two electrode testing cell (Teflon Swagelok®) and $1 \mathrm{M} \mathrm{H}_{2} \mathrm{SO}_{4}$ as electrolyte. The capacitance per active material 
of an electrode was obtained from charge-discharge experiments (C-D) in the range of 0.1-16 A g $\mathrm{g}^{-1}$ current density and with $\mathrm{U}=1$. Electrochemical impedance spectroscopy (EIS) analysis was also performed at $0 \mathrm{~V}$ (i.e. open circuit), over a frequency range of 1 $\mathrm{mHz}-100 \mathrm{kHz}$ and at an AC amplitude of $10 \mathrm{mV}$.

\section{Results and discussion}

The main reason for adding conductive additives when fabricating electrodes is to increase the electrical conductivity of the electrode. Figure 1 shows the effect of incorporating carbon black and graphene, using the two different methods described above. The addition of $\mathrm{CB}$ or GO increases the electrical conductivity in all cases, but the results are clearly different depending on whether they are added by physical blending or incorporated into the synthesis of the xerogel. In fact, the latter method (i.e. AXCB and AXGO samples) leads to a greater increase in electrical conductivity than physical mixing.

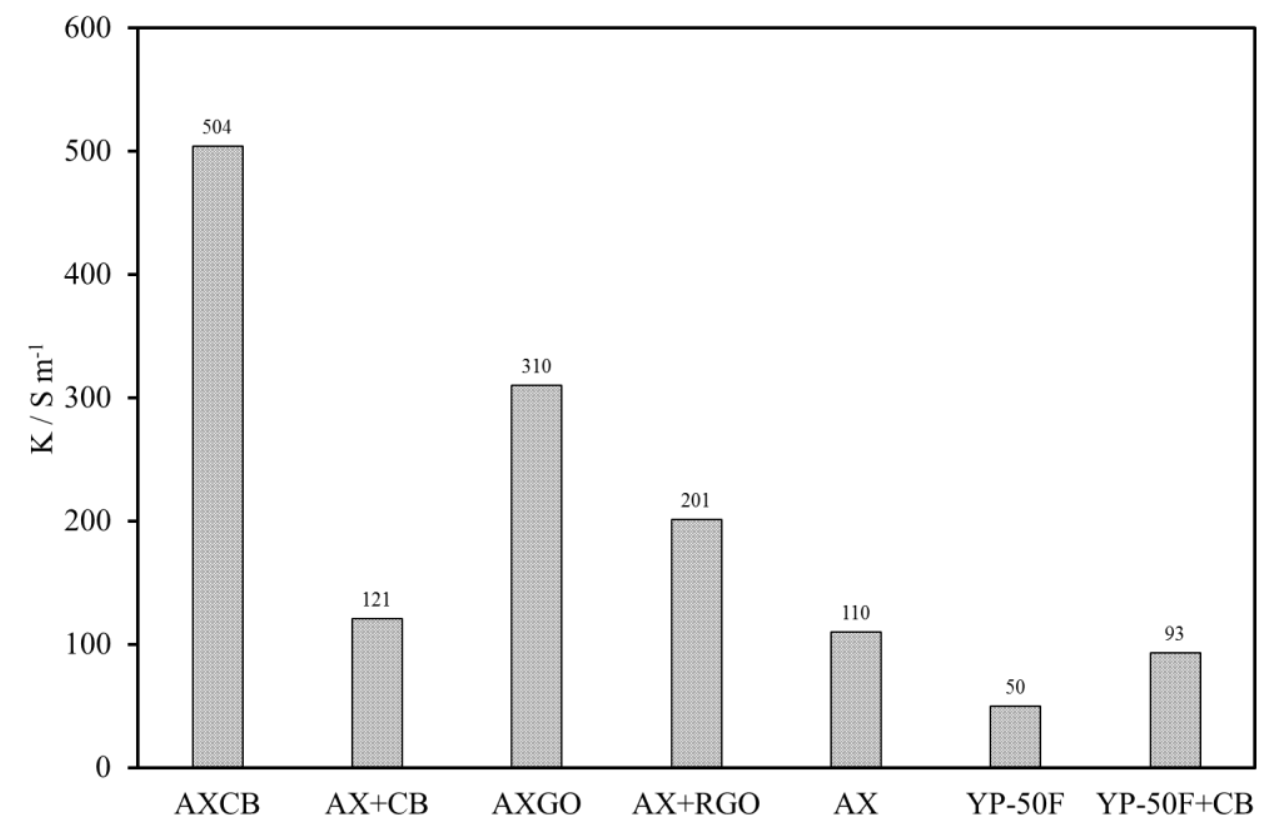


Figure 1. Electrical conductivity of the electrodes as evaluated by the four-point probe procedure.

The physical addition of $\mathrm{CB}$ results in a small increase in the electrical conductivity of $\mathrm{AX}$, but when $\mathrm{CB}$ is integrated within the polymeric structure, a sample with a high electrical conductivity and large surface area is obtained (i.e. ca. $500 \mathrm{~S} \mathrm{~m}^{-1}$ and $1600 \mathrm{~m}^{2}$ $\left.\mathrm{g}^{-1}\right)$

The performance of the different materials as electrodes in supercapacitors is shown in Figure 2. For the discussion of the different results obtained, two regions will be defined: (i) low current densities $\left(<1 \mathrm{~A} \mathrm{~g}^{-1}\right)$ where the maximum capacitance of the samples is obtained, and (ii) high current densities (1-16 $\left.\mathrm{A} \mathrm{g}^{-1}\right)$ where the operating conditions reveal most differences between the samples.

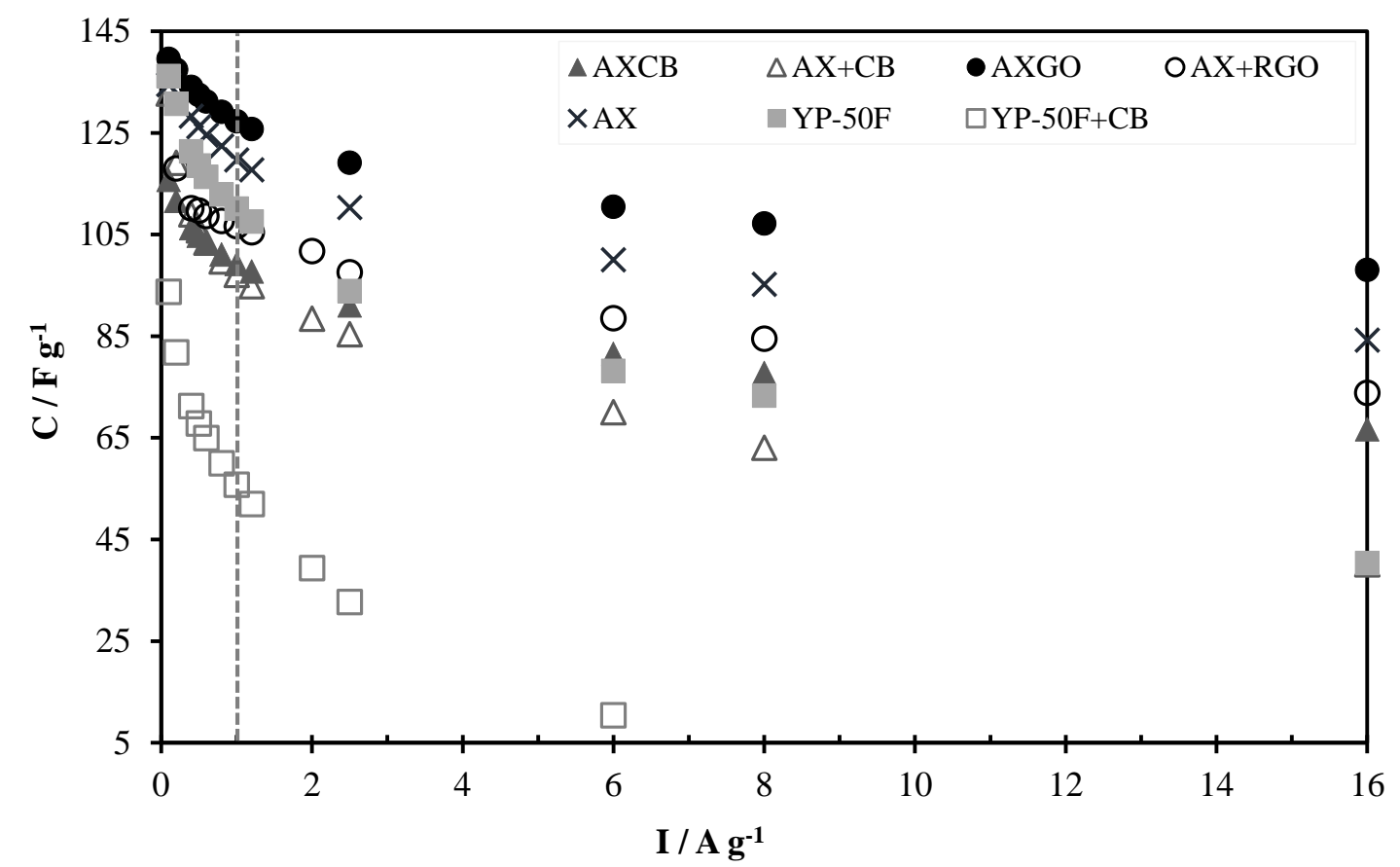

Figure 2.Capacitance variation of the samples studied in the current density range of $0.1-16 \mathrm{~A} \mathrm{~g}^{-1}$. 
At low current densities (i.e. $<1 \mathrm{~A} \mathrm{~g}^{-1}$ ), although all the samples have the same BET surface area (i.e. $1600 \mathrm{~m}^{2} \mathrm{~g}^{-1}$ ) and the same volume of micropores (i.e. $0.6 \mathrm{~cm}^{3} \mathrm{~g}^{-1}$ ), while two maximum capacitance values are observed: ca. $120 \mathrm{~F} \mathrm{~g}^{-1}$ for the $\mathrm{AX}+\mathrm{RGO}$, $\mathrm{AX}+\mathrm{CB}$ and $\mathrm{AXCB}$ samples, and ca. $140 \mathrm{~F} \mathrm{~g}^{-1}$ for the other samples (i.e. AXGO, AX and the reference YP-50F). It is clear that capacitance depends on something else but the micropore volume.

It is well known that (i) the pore size distribution determining the accessibility of the electrolyte to the micropores, (ii) the surface chemistry influencing the pseudocapacitance and the wettability of the electrode, and (iii) the electrical conductivity, are the relevant variables to take into account $[1,9-11]$.

Given the very high electrical conductivity of the AXCB sample (see Figure 1), this could be expected to have a high capacitance. However the results do not confirm that prediction. Moreover, the commercial sample that exhibits the lowest electrical conductivity (i.e. $50 \mathrm{~S} \mathrm{~m}^{-1}$ ) of the samples studied, shows very high capacitance values at low current densities. Consequently, other variables involved in the electrochemical process must be taken into account (i.e. diffusional or chemical factors).

Figure $3 \mathrm{a}$ shows the mesopore size distribution of the samples studied. It can be observed that physical mixing with the conductive additives (i.e. $\mathrm{AX}+\mathrm{CB}$ and AX+RGO) does not alter the mesopore size distribution of AX. However, the incorporation of the conductive additives within the polymeric structure of the xerogel (i.e. AXCB and AXGO) clearly causes the mesopores to widen, probably because the polymeric structure has been modified [7, 12]. 
(a)

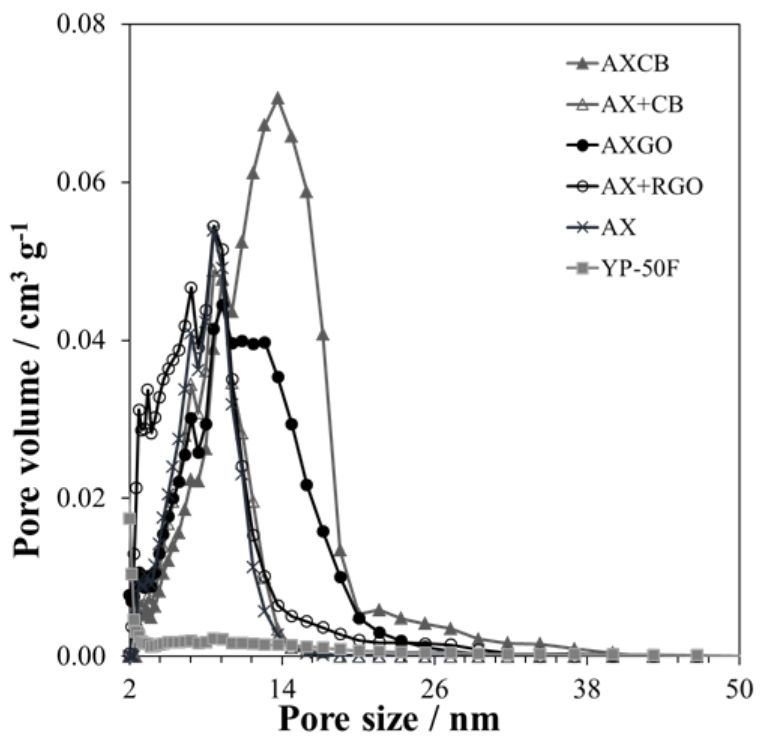

(b)

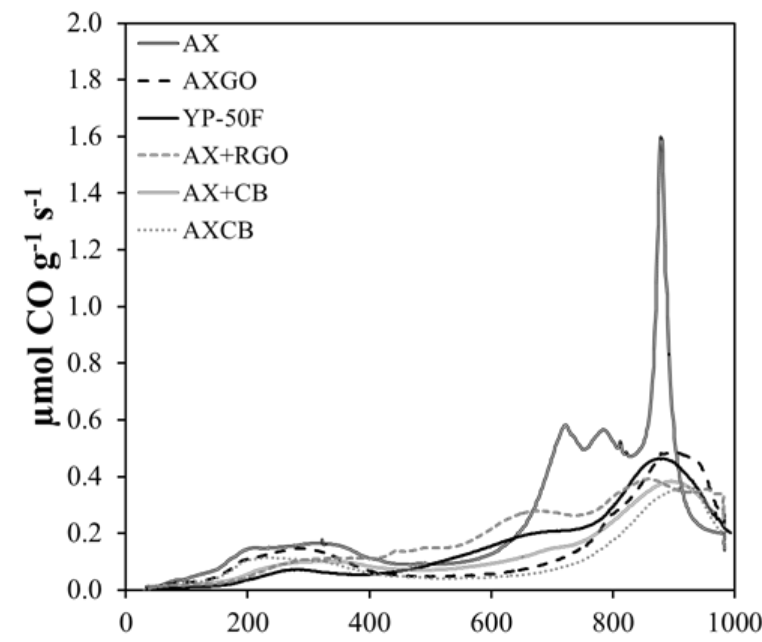

(c)

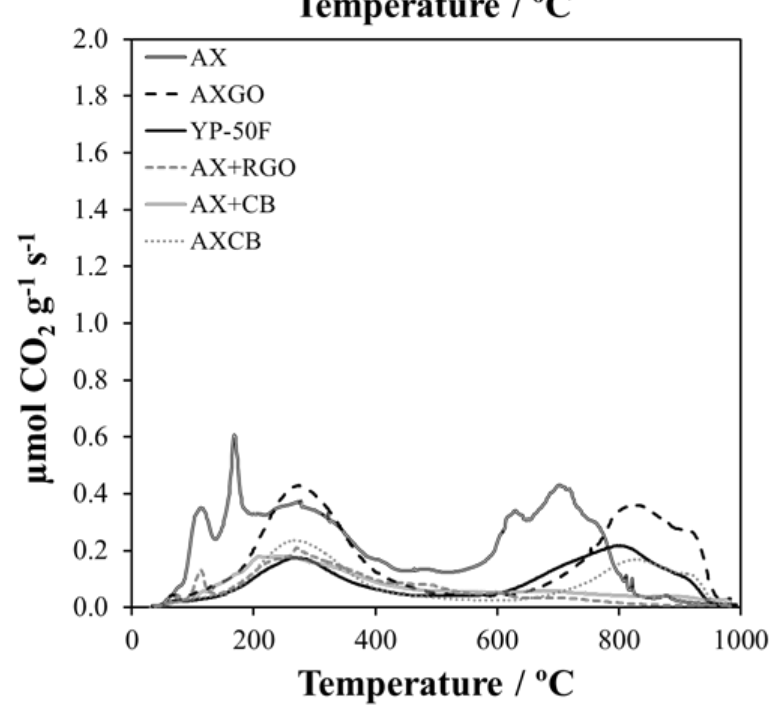

Figure 3. Mesopore size distributions (a), $\mathrm{CO}$ (b) and $\mathrm{CO}_{2}$ (c) temperature programmed desorption profiles of the samples studied. 
AXCB also has a suitable pore size distribution for the diffusion of electrolyte. The reason for its bad performance therefore must be its surface chemistry. TPD analysis reveals notable differences between samples. Figure $3 \mathrm{~b}$ and $3 \mathrm{c}$ shows that samples $\mathrm{AX}$ and $\mathrm{AXGO}$ present higher $\mathrm{CO}$ and $\mathrm{CO}_{2}$ evolution profiles than the rest of samples, indicating a higher content of oxygen functionalities. Regardless the type of oxygen functionalities present in these samples, it is clear that they have high oxygen content (i.e. 6.2 and $4.5 \mathrm{wt} \%$, respectively), that will influence on the pseudo-capacitance contribution (as was detected in previous work [13]) besides facilitating the wettability of these samples. On the contrary, it is worth to note the low $\mathrm{CO}$ and $\mathrm{CO}_{2}$ evolution of $\mathrm{AXCB}$ and $\mathrm{AX}+\mathrm{CB}$ samples, showing oxygen content of $2.7 \mathrm{wt} \%$, and indicating the very low concentration of oxygen functionalities, which would determine their probable hydrophobic character. The other two samples (i.e. AX+RGO and the reference material YP-50F) present intermediate oxygen content (i.e. 3 and $3.1 \mathrm{wt} \%$, respectively), which is not enough for showing a clear pseudo-capacitance contribution but it would improve their interaction with the aqueous electrolyte. In sum, it seems that at low current densities, surface chemistry is a relevant factor for a good performance in capacitance.

In the range of high current densities the differences between the samples become even greater (Figure 2). At these intensive conditions the supercapacitors charge faster, so carbons with a high electrical conductivity and with an appropriate pore size distribution (i.e. narrow mesopores) are, in principle, the most suitable.

Despite the fact that all the additives increase the electrical conductivity of the samples, only AXGO shows improvement with respect to AX over the whole range of current densities studied. AXGO displays the best performance with a capacitance of $98 \mathrm{~F} \mathrm{~g}^{-1}$ at $16 \mathrm{~A} \mathrm{~g}^{-1}$ that represents an enhancement of $16 \%$ and $143 \%$ with respect to AX and YP50F, respectively. Moreover, it is also the most stable sample with a capacitance drop of 
only $30 \%$ between 0.2 and $16 \mathrm{~A} \mathrm{~g}^{-1}$. AX+RGO and AXCB also show good stability with charge retention of ca. $60 \%$, due to their mesoporous structure and their good conductivity values. However, it is worth mentioning that $\mathrm{CB}$, although it shows excellent values in terms of electrical conductivity $\left(1700 \mathrm{~S} \mathrm{~m}^{-1}\right)$ and surface area (1600 $\mathrm{m}^{2} \mathrm{~g}^{-1}$ ) has very poor properties for its use in supercapacitors, since its addition during the synthesis $(\mathrm{AXCB})$ or post-synthesis $(\mathrm{AX}+\mathrm{CB})$ or even its addition to the commercial sample (see sample YP-50F+CB in Figure 2) lead to a very poor performance.

In order to explain these results, the electrochemical impedance performance of the xerogel samples was also evaluated, the results of which are shown in Figure 4.

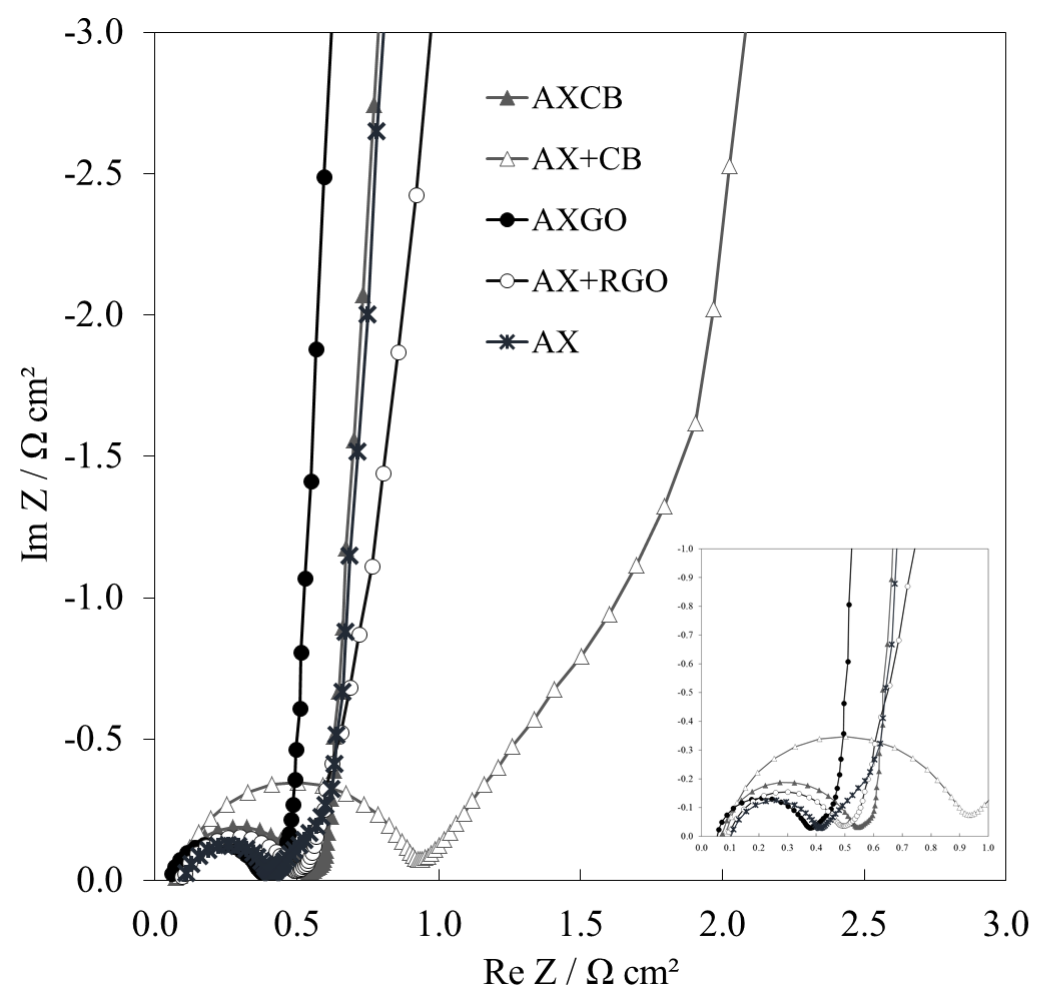

Figure 4. Nyquist plot of the carbon xerogels studied.

It can be observed that the two samples with the lowest electrical conductivity values $\left(\mathrm{AX}+\mathrm{CB}\right.$ and $\mathrm{AX}$, see Figure 1) have a more well-defined $45^{\circ}$ Warburg zone, which 
implies a higher mass transfer control probably due to the lower polarization of the surface during the charge process. The equivalent series resistance (ESR) determined as the intersection of the semi-circle at high frequencies with the real axis [14], shows that, although $\mathrm{CB}$ is a very good conductive material, $\mathrm{AX}+\mathrm{CB}$ and $\mathrm{AXCB}$ are the most resistive samples (i.e. widest semi-circles) recording values of 0.9 and $0.5 \Omega \mathrm{cm}^{2}$, respectively. The porous structure of these samples does not seem to produce diffusional problems. In this sense the sample $\mathrm{AX}+\mathrm{CB}$ displays the worse results because its relativity low electrical conductivity (see Figure 1), besides a poor wettability due to its poor surface chemistry. The best global performance (i.e. less defined Warburg zone and narrowest semi-circle) is displayed by the AXGO sample, which has the smallest Warburg zone and ESR.

\section{Conclusions}

It can be concluded that although increasing the electrical conductivity of the electrodes is necessary for improving supercapacitor performance, other fundamental characteristics for optimum electrochemical behavior in supercapacitors must be taken into account. The incorporation of the additive within the structure of the active material causes a notable improvement in the performance of the electrodes over the whole range of current densities. Thus, the incorporation of graphene oxide within the carbon xerogel structure during the synthesis seems to be the best way to obtain an electrode with superior capacitance performance and stability.

\section{Acknowledgements}


The authors gratefully acknowledge the financial support received from the Ministerio de Economía, Industria y Competitividad from Spain (Project CTQ2017-87820-R). MCR also acknowledges the support from CSIC (Project I.E. 201880E010).

\section{References}

[1] F. Beguin, V. Presser, A. Balducci, E. Franckowiak, Carbons and electrolytes for advanced supercapacitors, Adv. Mater. 26 (2014) 2219-2251.

https://doi.org/10.1002/adma.201304137.

[2] E.G. Calvo, N. Ferrera-Lorenzo, J.A. Menéndez, A. Arenillas, Microwave synthesis of micro-mesoporous activated carbon xerogels for high performance supercapacitors, Microporous and Mesoporous Materials, 168 (2013) 206-212. https://doi.org/10.1016/j.micromeso.2012.10.008.

[3] B.E. Conway, Eelectrochemical supercapacitors: scientific fundamentals and technological applications, Springer Science \& Business Media, 2013.

[4] E.G. Calvo, E.J. Juárez-Pérez, J.A. Menéndez, A. Arenillas, Fast microwaveassisted synthesis of tailored mesoporous carbon xerogels, Journal of Colloid and Interface Science, 357 (2011) 541-547. https://doi.org/10.1016/j.jcis.2011.02.034.

[5] N. Rey-Raap, E.G. Calvo, J.M. Bermúdez, I. Cameán, A.B. García, J.A. Menéndez, A. Arenillas, An electrical conductivity translator for carbons, Measurement: Journal of the International Measurement Confederation, 56 (2014) 215-218.

http://dx.doi.org/10.1016/j.measurement.2014.07.003.

[6] L. Song, F. Khoerunnisa, W. Gao, W. Dou, T. Hayashi, K. Kaneko, M. Endo, P.M. Ajayan, Effect of high-temperature thermal treatment on the structure and adsorption properties of reduced graphene oxide, Carbon 52 (2013) 608-612. https://doi.org/10.1016/j.carbon.2012.09.060.

[7] M. Canal-Rodríguez, A. Arenillas, N. Rey-Raap, G. Ramos-Fernández, I. MartínGullón, J.A. Menéndez, Graphene-doped carbon xerogel combining high electrical conductivity surface area for optimized aqueous supercapacitors, Carbon, 118 (2017) 291-298. https://doi.org/10.1016/j.carbon.2017.03.059.

[8] E.G. Calvo, F. Lufrano, P. Staiti, A. Brigandì, A. Arenillas, J.A. Menéndez, Optimizing the electrochemical performance of aqueous symmetric supercapacitors based on an activated carbon xerogel, J Power Sources, 241 (2013) 776-782. https://doi.org/10.1016/j.jpowsour.2013.03.065. 
[9] A.G. Pandolfo, A.F. Hollenkamp, Carbon properties and their role in supercapacitors, J Power Sources, 157 (2006) 11-27.

https://doi.org/10.1016/j.jpowsour.2006.02.065.

[10] C. Lin, J.A. Ritter, B.N. Popov, Development of carbon-metal oxide supercapacitors from sol-gel derived carbon-ruthenium xerogels, Journal of the Electrochemical Society, 146 (1999) 3155-3160. http://dx.doi.org/10.1149/1.1392448.

[11] X. Li, B. Wei, Supercapacitors based on nanostructured carbon, Nano Energy, 2 (2013) 159-173. http://dx.doi.org/10.1016/j.nanoen.2012.10.004.

[12] K. Guo, H. Song, X. Chen, X. Du, L. Zhong, Graphene oxide as an anti-shrinkage additive for resorcinol-formaldehyde composite aerogels, Physical Chemistry Chemical Physics, 16 (2014) 11603-11608. http://dx.doi.org/10.1039/C4CP00592A.

[13] M. Canal-Rodríguez, J.A. Menéndez, M.A. Montes-Morán, I. Martín-Gullón, J.B. Parra, A. Arenillas, The role of conductive additives on the performance of hybrid carbon xerogels as electrodes in aqueous supercapacitors, Electrochimica Acta, 295 (2019) 693-702. https://doi.org/10.1016/j.electacta.2018.10.189

[14] A.B. Fuertes, M. Sevilla, Superior capacitive performance of hydrochar-based porous carbons in aqueous electrolytes, ChemSusChem, 8 (2015) 1049-1057.

https://doi.org/10.1002/cssc.201403267. 